\title{
Effect of Electrode Manufacturing Defects on Electrochemical Performance of Lithium-Ion Batteries: Cognizance of the Battery Failure Sources
}

D. Mohanty, ${ }^{\mathrm{a},{ }^{*}}$ E. Hockaday, ${ }^{\mathrm{b}} \mathrm{J}_{\mathrm{L}} \mathrm{Li},{ }^{\mathrm{a}}$ D. K. Hensley, ${ }^{\mathrm{c}}$ C. Daniel, ${ }^{\mathrm{a}, \mathrm{d}}$ and D. L. Wood III ${ }^{\mathrm{a} d, *}$

${ }^{a}$ Oak Ridge National Laboratory, Energy \& Transportation Science Division, One Bethel Valley Road, P.O. Box 2008, Oak Ridge, Tennessee, U.S.A.

${ }^{\mathrm{b}}$ Department of Electrical Engineering, University of Tennessee, Knoxville, Tennessee, U.S.A.

${ }^{c}$ University of Tennessee, Bredesen Center for Interdisciplinary Research and Graduate Education, 418 Greve Hall, 821 Volunteer Blvd., Knoxville, Tennessee, U.S.A.

${ }^{\mathrm{d} C e n t e r}$ for Nanophase Materials Sciences, Oak Ridge National Laboratory, One Bethel Valley Road, Oak Ridge, Tennessee, U.S.A.

* mohantyd@ornl.gov (DM) and wooddl@ornl.gov (DLW)

\begin{abstract}
:
During LIB electrode manufacturing, it is difficult to avoid the certain defects that diminish LIB performance and shorten the life span of the batteries. This study provides a systematic investigation correlating the different plausible defects (agglomeration/blisters, pinholes/divots, metal particle contamination, and non-uniform coating) in a $\mathrm{LiNi}_{0.5} \mathrm{Mn}_{0.3} \mathrm{Co}_{0.2} \mathrm{O}_{2} / \mathrm{NMC532}$ positive electrode with its electrochemical performance. In addition, an infrared thermography technique was demonstrated as a nondestructive tool to detect these defects. The findings show that cathode agglomerates aggravated cycle efficiency, and resulted in faster capacity fading at high current density. Electrode pinholes showed substantially lower discharge capacities at higher current densities than baseline NMC 532electrodes. Metal particle contaminants have an extremely negative effect on performance, at higher $C$-rates. The electrodes with more coated and uncoated interfaces (non-uniform coatings) showed poor cycle life compared with electrodes with fewer coated and uncoated interfaces. Further, microstructural investigation provided evidence of presence of carbon-rich region in the agglomerated region and uneven electrode coating thickness in the coated and uncoated interfacial regions that may lead to the inferior electrochemical performance. This study provides the importance of monitoring and early detection of the electrode defects during LIB manufacturing processes to minimize the cell rejection rate after fabrication and testing.
\end{abstract}




\section{Introduction}

Highly efficient, cost-effective energy-storage devices are needed for plug-in electric vehicles (PHEVs) to compete with vehicles powered by internal combustion engines.[1, 2] Lithium-ion battery (LIB) technology has proven its superiority over all other energy storage technologies in powering consumer electronics and is the leading candidate for implementation in electric vehicles $(\mathrm{EVs}) .[3,4]$ Several efforts have been made to enhance the performance of current LIB systems to meet the needs of EVs as per the US Advanced Battery Consortium LLC, requirements.[5, 6]

In addition, the cost for a PHEV battery should be reduced to $\$ 125 / \mathrm{kWh}$, and the power density should be increased simultaneously from 400 to 2000W/L by 2022.[6] Reducing the LIB manufacturing cost remains a challenge in the current LIB industries, $[7,8]$ and detailed attention to fundamental and applied science is required. Strategies for overcoming manufacturing costs include implementing low-cost processing and adequate quality control (QC) tools to reduce the scrap rate in larger format batteries.[9-12]

Our group at Oak Ridge National Laboratory has demonstrated that adapting aqueous processing instead of N-methylpyrrolidone (NMP) processing for cathode coating (slurry) mixing could reduce the battery cost by $10 \%$ per watthour. $[13,14]$ In addition, we recently reported the implementation of QC techniques on a slot-die coating instrument to reduce the scrap rates in larger format cells associated with LIB manufacturing.[15] A laser caliper system was mounted on the slot-die coating line to monitor the wet thickness of the coated electrodes (inhomogeneity in electrode thickness results in variation in electrode balance locally and affects the state of charge during cell test ). An infrared (IR) thermography technique was also utilized to detect very small flaws in the dried electrodes that might hamper the performance of LIBs (flaws generated 
during coating that are not visible to the unaided eye and not with the aid of an optical microscope).[15] The motivation behind implementing IR thermography was that, if electrode flaws and contaminants could be detected in- line near the particular processing steps that generate them, then the electrode material could be marked as unusable, and the processing protocol could be adjusted to eliminate the defects timely. The defective electrodes can be discarded ahead of the cell manufacturing steps and thus could reduce the cell scrap rate in larger format cells significantly.

In addition to the QC tools $[11,12,15]$ an understanding of correlations between the flaws and the electrode's performance are indispensable to establishing pass/fail criteria for the electrodes. In this report, we present a thorough investigation of the correlations between the electrochemical performance of LIBs and the different defects in the LIB positive electrodes. Further, microstructural analyses of non-defective baseline (baseline) and defective electrodes were carried out to obtain some insights into the mechanisms behind the detrimental influence of the defects. To accomplish this, various types of defects were generated on a Ni-Mn-Co based positive electrode $\left(\mathrm{LiNi}_{0.5} \mathrm{Mn}_{0.3} \mathrm{Co}_{0.2} \mathrm{O}_{2}\right.$ or NMC 532), and the electrode underwent electrochemical cycling. We chose "NMC 532" as the positive electrode material because it is a promising cathode material for EV application.[16] This work addressed the following important scientific questions:

i) What are the defects that might be created during the electrode manufacturing processes?

ii) What is the effect of those defects on LIB charging and discharging cycles?

iii) How do the defects alter the coulombic efficiency, power (rate capability) and lifetime (cycle life) of LIBs? 
iv) Are any microstructural changes responsible for the diminished performance of an LIB manufactured with the defective NMC 532 electrodes?

\section{Experimental}

Materials and cell assembly: NMC 532 powders were purchased from Toda America Inc.. The electrodes were fabricated at the U.S. Department of Energy (DOE) Battery Manufacturing R\&D facility at Oak Ridge National Laboratory. Active NMC 532 powder (90 wt \%), Polyvinylidene fluoride (Solvay 5130, 5 wt \%), and carbon black (Denka, 5 wt \%) were dispersed in N-Methyl-2pyrrolidone with a planetary mixer (Ross PDM-1/2). A cathode was fabricated by applying the slurry to one side of a $15 \mu \mathrm{m}$ thick Al foil with a slot-die coater (Frontier Industrial Technology, Inc.). The wet coating thickness was $118.5 \mu \mathrm{m}$, and the solid loading was $15 \mathrm{mg} / \mathrm{cm}^{2}$. The anode was fabricated using A12 natural graphite (ConocoPhillips, A12) with A12 graphite, PVDF (Kureha 9300), and super P Li in 92/6/2 wt\%. The anode loading is $8.0 \mathrm{mg} / \mathrm{cm}^{2}$ and the electrode balance is $1.1(\mathrm{~N} / \mathrm{P})$ when coupled with the NMC532 cathode.

Full coin cells were built from uncalendared electrodes. The cells were assembled by using 0.5 inch diameter disks of the NMC 532 cathode and the A12 graphite anode, and 0.55 inch diameter Celgard 2325 separator. Defect-free cathode material was used to fabricate baseline specimens; test specimens were fabricated from defective cathode material (see the "Results" section for a description of the different types of defects that are considered for this study). A 1.2 M solution of $\mathrm{LiPF}_{6}$ in ethylene carbonate (EC)-ethyl methyl carbonate (EMC) (3:7 ratio by weight) (BASF®) was used as the electrolyte. Electrochemical cycling was carried out by using a BioLogic VSP potentiostat controlled with EC-Lab software (V. 10.02).

Electrochemical testing: The assembled full coin cells were tested for rate performance and cyclelife testing. Rate performance tests were carried out in the voltage window of 2.2 to $4.2 \mathrm{~V}$ at 
$0.05 \mathrm{C}, 0.1 \mathrm{C}, 0.2 \mathrm{C}, 1 \mathrm{C}, 2 \mathrm{C}, 5 \mathrm{C}$ (where $1 \mathrm{C}=160 \mathrm{~mA} / \mathrm{g}$ ). The cycle life tests were performed at high $C$ - rate $(0.5 \mathrm{C} /-2 \mathrm{C}$, and $0.5 \mathrm{C} /-5 \mathrm{C})$. For calculating statistics and error, five representative full coin cells from each baseline and defective electrodes were considered for every electrochemical experiment. The error in each data point was computed based on the T-distribution formula with a degree of freedom of 4 and a $95 \%$ confidence limit.

IR thermography: IR thermography was employed on the NMC 532 electrodes to demonstrate its value as a non-destructive evaluation (NDE) technique. We used an FLIR SC-8200 IR camera with a $25 \mathrm{~mm}$ lens (no filters or extender rings) and a 6000 Joule Hensel flash system at $\sim 60 \%$ flash power. The camera has a resolution of 1024 by 1024 pixels and a wavelength range of 3 to 5 $\mu \mathrm{m}$. Line scans were performed to obtain the emissivity results from both defective and baseline elctrodes.

Microstructural Characterization: Cells were disassembled for microstructural analysis after 0.5C/-2C cycle-life testing. Scanning electron microscope (SEM) images were obtained in a Carl Zeiss Merlin SEM operating at $15 \mathrm{kV}$ for imaging and energy-dispersive spectroscopy (EDS). The EDS results were obtained with a system from Bruker Nano GmbH using an XFlash 5030 detector. The cross-sectional images were collected by a Hitachi S3400 SEM operated at $10 \mathrm{kV}$.

\section{Results:}

A. Generation of different types of defects: Different types of defects figure $1(\mathrm{a}, \mathrm{c}, \mathrm{e}, \mathrm{h})$ that were artificially introduced during electrode-coating process is discussed in this section.

i) Blisters/agglomerates (Figure 1a) can be generated if the components in the coating have not been mixed for appropriate required time to form homogeneous slurry and/or deviating the slurry-feeding rate. Agglomerates of inactive components such as binders and carbon black 
can lead to higher inactive component content and lower the gravimetric capacity of the test electrode. The agglomeration in this experiment was induced by changing the slurry feeding rate. The area of agglomerated regions in this experiment was $\sim 4.67 \times 10^{5} \mu \mathrm{m}^{2}$ (electrode area is $1270 \times 10^{5} \mu \mathrm{m}^{2}$ and the diameter of the circular disc electrodes are $\left.6350 \mu \mathrm{m}\right)$.

ii) Divots/Pinholes (figure 1c) are location where coating is missing, often caused by captured bubbles in slurry. They reduce the amount of active material and can expose the current collector to the electrolyte and thus may decrease the electrochemical capacity. The pinholes were generated by applying a very small NMP to the coating to remove the active materials from the current collector. The area of pinhole regions ( consisted of approximately 10 small pinholes) in this experiment was $\sim 8.6 \times 10^{6} \mu \mathrm{m}^{2}$ (electrode area is $1270 \times 10^{5} \mu \mathrm{m}^{2}$ and the diameter of the circular disc electrodes are $\left.6350 \mu \mathrm{m}\right)$.

iii) Metal particle contaminants (figure 1e) can be inadvertently introduced during the coating process, either from hardware or from other sources. We tested Al foil and Co-powder contaminants in this study. During the coating process, small pieces $(\leq 2 \mathrm{~mm})$ of $\mathrm{Al}$ foil were placed on the wet coating or Co powder $(\leq 0.01 \mathrm{~g})$ of was sprinkled on different areas on the wet coating to emulate metal particle contamination. The motivation behind using excessive amount of contaminants was to observe the explicit effect on electrochemistry.

iv) Exaggerated non-uniform coating (figure 1g) can result if the slurry-feeding tool has a serious malfunction or if inaccurate mixing protocols are followed. This type of defect clearly has coating missing from several areas on the cathode material. Our goals were to understand the correlation between these defects and electrochemical performance and to obtain an understanding of the interfacial effect (the number of interfaces between coated and uncoated regions) of a given type of defect on electrochemical performance. We designed a special shim for slot-die coating to introduce a $6 \mathrm{~mm}$ wide area (a $1 \times$ big 
defective area) and a $2 \mathrm{~mm}$ wide area (a $3 \times$ small defective area) where the coating was missing (See below in this section).

IR thermography: The IR thermography was employed to demonstrate as QC technique and to investigate the change in emissivity from the defective regions in the NMC 532 electrodes. Figure $1(\mathrm{~b}, \mathrm{~d}, \mathrm{f}, \mathrm{h})$ present the corresponding IR thermography images of NMC 532 electrodes with various types of defects. Depending on the nature of the defects, the emissivity decreases or increases. For example, emissivity increases across the defect region that correspond to a blister or agglomerate (Figure 1a, b) but an emissivity decrease corresponds to pinholes, exaggerated non-uniform coating, and metal contaminants (Figure $1 \mathrm{c}, \mathrm{d} ; \mathrm{e}, \mathrm{f} ; \mathrm{g}, \mathrm{h}$ ). In the case of agglomerates, heat cannot be released as quickly. Therefore, emissivity increases. In the case of pinholes and metal particle contaminants, and exaggerated non-uniform coatings where heat is released from the coating surface more quickly, emissivity decreases. These results show the capability of IR thermography as an NDE-QC method to distinguish between different types of defects based on increase and decrease in the emissivity values.

\section{B. Effect of defects on electrochemical performance}

i) Rate capability and coulombic efficiency:

Figure 2 shows the comparison of gravimetric $(\mathrm{mAh} / \mathrm{g}$ ), and full capacity (mA.h) ( Figure 2a), and the coulombic efficiency (Figure 2b) at different current densities between an NMC 532 electrode having agglomeration defects and the baseline (non-defective) NMC 532 electrode. Surprisingly, both the gravimetric capacity and full cell capacity in case of defective electrode are relatively higher than they are for the baseline electrode; however, the coulombic efficiency for the agglomerated electrode is lower than that of the baseline electrode. The reasons for improved rate capability in case of agglomerated electrode are currently unknown and demands further 
detailed investigation. Nonetheless, the result indicates that electrode agglomerations have a negative impact on the coulombic efficiency. The reason for divergent electrochemical results in the case of the electrode having agglomerates may be due to the presence of excess inactive binder and carbon black in the defective electrodes. The inactive components may induce insufficient electrical connection or bad mechanical fixation resulting in lower coulombic efficiency. Figure $2 \mathrm{c}$ and $\mathrm{d}$ present comparisons between the rate capability and the coulombic efficiency of the baseline electrodes and the electrodes with pinholes. The gravimetric and fullcapacity values for the defective electrode are smaller than the values from the baseline electrode. Interestingly, at higher current density (1C, and 2C), the capacity for the electrodes having pinhole defects decrease $\sim 63 \%$ as compared to the baseline electrode. At higher $C$-rate $(1 \mathrm{C}, 2 \mathrm{C}$, and $5 \mathrm{C}$ ), the coulombic efficiency is lower for the electrode having pinholes than it is for the baseline electrode.

Figure 3 presents the comparison of rate capability and coulombic efficiency from the baseline NMC 532 electrodes with electrodes having an exaggerated non-uniform coating (a, b), Co particle contaminants (c, d), and $\mathrm{Al}$ foil contaminants (e, f). From figure $3 \mathrm{a}$, it is evident that the gravimetric capacity for the exaggerated non-uniform electrode is $10 \%$ to $20 \%$ less (especially at higher $C$ rate) than that of the baseline electrode; however, the full cell capacity for the defective electrode is $60 \%$ to $70 \%$ lower than the capacity of the baseline electrode. This indicates there is less active material in the electrode. The coulombic efficiency at different current rates for the exaggerated non-uniform electrode is slightly less than that of the baseline electrode. From Figures $3 \mathrm{c}$ and $\mathrm{d}$, it is clear that the presence of metal (Co) contaminants decreases the gravimetric and full capacity and that it has a detrimental effect on coulombic efficiency. Surprisingly several cells failed to deliver capacity at high rate (2C and $5 \mathrm{C}$ discharge rate). The poor electrochemical performance may be due to the (1) participation of metal particles in the 
electrochemical reactions forming metallic alloys that impede the lithium diffusion paths for the lithiation and delithiation reactions and eventually lowers the capacity and/or, (2) because the Co particles are smaller than the pore size of the separator, they might diffuse through the separator pores and create a short circuit in the LIB cell that eventually leads to cell failure. The sudden failure of the cells at higher rate indicates the short circuit in the LIB cells due to diffusion of the small Co-particles through the separator. In the case of Al-foil contaminants, the discharge capacity is lower than that of the baseline electrodes at $2 \mathrm{C}$ (Figure 3e); however, the coulombic efficiency is significantly lower for the electrode contaminated with Al foil (Figure 3f). The results from the rate capability test and efficiency evaluations can be summarized as follows:

- The presence of agglomerates in the positive electrode coating decreases the cell coulombic efficiency.

- The introduction of pinholes in the positive electrode coating lowers the coulombic efficiency and results in poor rate capability, especially at high current density.

- An exaggerated non-uniform coating shows poor rate capability.

- Metal particle contaminants may introduce a soft short circuit and therefore may significantly reduce capacity of the cell.

It is noted that the case addressed here is simplified and the actual case could be much more complex. For example, there could be more than one type of defect in the electrode. In addition, to match closely to application, same A12 anode was used for full cell assembly with baseline and defective electrodes. The electrode balance varies in those cells, which is another factor possibly affecting the performance, e.g. coulombic efficiency. To obtain a better understanding of the effect of a non-uniform coating on electrode performance, a special shim was designed for the slot-die coater (Figure 4a). The die produces two areas of exaggerated non-uniformity (i.e., 
grooves where the coating is missing from an otherwise uniformly coated surface): a single $6 \mathrm{~mm}$ wide groove (the $1 \mathrm{X}$ big defective area) and three parallel $2 \mathrm{~mm}$ wide grooves (the $3 \mathrm{X}$ small defective area). Corresponding optical and IR thermography images of electrode material produced by using that shim set are presented in Figure 4 b-c. Figures $4 d-g$ also shows comparisons of the performance of the baseline electrode with the performance of the $1 \mathrm{X}(\mathrm{d}, \mathrm{e})$ and $3 \mathrm{X}$ electrodes (f, g). The IR thermography image shows the contrast in the emissivity values in the uncoated and coated areas. It was found that cells fabricated with non-uniformly coated electrodes were not able to deliver capacity at a high rate. The coulombic efficiency shows a similar trend for both the electrode having the $1 \mathrm{X}$ big defect and the electrode having the $3 \mathrm{X}$ slaa defects.

ii) Cycle life (capacity-fade) test at 2C and 5C:

The discharge capacity fading tests for the defective NMC 532 electrodes were performed for 200 cycles at two different conditions, at $2 \mathrm{C}$ and $5 \mathrm{C}$ (the charge rate was $0.2 \mathrm{C}$ ), and the results were compared with the data from testing the baseline electrode. Electrodes with the four different types of defects were chosen for analysis. In Figure 5, the gravimetric discharge capacity during the 200 charge-discharge cycles are plotted for the baseline and defective electrodes. The gravimetric capacity and full capacity before and after 200 cycles (figure 6a-b), and capacity retention after 200 cycles at $2 \mathrm{C}$ and $5 \mathrm{C}$ rates are presented in Figure $6 \mathrm{c}$. The performance data from metal particle contamination was not included in figure $6 c$, because of the severe detrimental behavior after very less charge-discharge cycles.

a) Electrode agglomerates

Faster discharged capacity degradation 
Figures 5a and $\mathrm{b}$ show the capacity-fading results from the baseline electrode and the electrode having agglomerates. Corresponding gravimetric and full-capacity values before and after 200 cycles at $2 \mathrm{C}$, and $5 \mathrm{C}$ is presented in figure 6a-b. Approximately $70 \%$ of full/gravimetric capacity was retained after 200 cycles at $2 C$ for the baseline electrode, whereas for the electrode containing agglomerates, $12 \%$ of discharge capacity was retained after 200 cycles. At the $5 C$ rate, the baseline electrode showed $\sim 50 \%$ discharge capacity retention after 200 cycles. In contrast, discharge capacity retention of $\sim 14 \%$ was observed for the electrodes with agglomerations in them.

b) Electrode pinholes

\section{Less severe capacity degradation}

Figures $5 \mathrm{c}$ and d (corresponding gravimetric, and full-capacity before and after 200 cycles is presented in figure 6a-b) show the capacity-fading results for electrodes having pinholes compared with the baseline electrodes. In presence of pinholes, capacity fading is significant; however, it is much less severe to the fading caused by the agglomerates. The capacity retention was $47 \%$ and $40 \%$ after 200 charge-discharge cycles at the $2 \mathrm{C}$ and 5C rates, respectively. Fullcapacity retention was higher for electrodes having pinholes than it was for the electrodes having agglomerates.

c) Metal particle contaminants

\section{- Unstable cell performance at high-rate}

Figure 5 e shows the capacity-fade testing results for the electrode with Co metal particle contaminants compared with results for the baseline electrode. The results show zero capacity for the defective electrode after three cycles when operated at $2 \mathrm{C}$; the cell was not stable, and cell failure was observed at $5 \mathrm{C}$. Figure $5 \mathrm{f}$ shows the capacity-fade testing results for the electrode 
having $\mathrm{Al}$ foil contaminants. The results show that the capacity degradation was faster for the defective electrode than it was for the baseline electrode.

d) Non-uniform electrode coating with $1 \mathrm{X}$ defect and $3 \mathrm{X}$ defects

-Severe capacity degradation for electrodes having the $3 X$ defects

Figures $5 \mathrm{~g}$ and $\mathrm{h}$ show a comparison of the capacity-fading results for a baseline electrode with the results for an electrode having the $1 \mathrm{X}$ defect, at $2 \mathrm{C}$ and $5 \mathrm{C}$, respectively; Figure $5 \mathrm{i}$ and $\mathrm{j}$ show a comparison of the capacity-fading results for a baseline electrode compared with the results for an electrode having a $3 \mathrm{X}$ non-uniform coating, at $2 \mathrm{C}$ and $5 \mathrm{C}$, respectively. Figure $6 \mathrm{a}-\mathrm{b}$ shows the comparison of gravimetric and full capacity values before and after 200 cycles for $1 \mathrm{X}$ and $3 \mathrm{X}$ defects at 2C, and 5C discharge rate. Analysis showed that the capacity degradation rate was higher in electrodes having greater interfacial length (i.e., the $3 \mathrm{X}$ defect) than it was for electrodes having the smaller interfacial length (i.e., the $1 \mathrm{X}$ defect). At 5C, the gravimetric discharge capacity retention was only $7 \%$ after 200 cycles in the $3 \mathrm{X}$ defective electrode, whereas $45 \%$ full capacity retention was observed in the $1 \mathrm{X}$ electrode. The poor performance in $3 \mathrm{X}$ defective electrodes as compared to the $1 \mathrm{X}$ defective electrode may be due to the excessive delamination in case of $3 \mathrm{X}$ defective electrodes and due to the more interfaces between coated and uncoated areas (refer to the "Understanding the defect-electrochemistry-microstructure correlation" section below).

From figure 6 , it is summarized that the rate of capacity fading at high current rate (at $5 C$ ) is in the sequence of: non-defective baseline $<1 \mathrm{X}$ non-uniform coating < pinholes < agglomerates < $3 \mathrm{X}$ non-uniform coating, and at $2 C$ discharge current rate: non-defective baseline $<1 \mathrm{X}$ nonuniform coating $<$ pinholes $<3 \mathrm{X}$ non-uniform coating $<$ agglomerates. The electrodes having agglomeration shows little improvement in the capacity at $5 C$ discharge rate as compared to the $2 C$ discharge rate. This is not obvious, however the reason might be due to the limited sample size 
or the difference in the agglomeration size in the electrodes that was used for $2 \mathrm{C}$ experiment and $5 \mathrm{C}$ experiments.

In the next section, we present the microstructural investigations conducted to understand the relatively poor electrochemical performance in electrodes that have agglomerate defects and $3 \mathrm{X}$ non-uniform coatings.

\section{Understanding the defect-electrochemistry-microstructure correlation}

In Figure 7, the SEM micrographs and corresponding X-ray elemental mapping results are presented for a baseline NMC 532 electrode (Figure 7a) and for an electrode with agglomeration defects (Figure 7b). The SEM micrographs and the elemental mapping from the baseline electrode show the uniform distribution of the inactive components and spherical active particles. The images of the agglomerated electrodes clearly show evidence of phase-rich regions of inactive (carbon rich) material. Inhomogeneous distribution of active component was also observed near to the agglomerated region. The presence of large inactive component can cause insufficient electrical connection and/or bad mechanical fixation, resulting in capacity degradation (Figure $6 \mathrm{a}, \mathrm{b}$, and c). It is also anticipated that some agglomerates areas in the defective electrode may include active component. The presence of large active component agglomerates protrudes the surface causing an inhomogeneous electrical field distribution. Large agglomerates can also negatively affect the coating process by producing scratches in the electrode that might contribute to capacity degradation.

Cross-sectional SEM images of baseline electrodes and electrodes having non-uniform coatings were investigated to gain insights into their poor electrochemical performance (Figure 8). The SEM images reveal that the coating is uniform across the baseline electrodes. (Figures $8 \mathrm{a}-\mathrm{c}$ ). However, in the non-uniform coated electrodes, the interface between the coated and uncoated regions does not follow as the step function; rather, it maintains a slope. The interface has less 
active material and less contact between the coatings (Figures $8 \mathrm{~d}-\mathrm{h}$ ), and the current collector was lost in some regions, as depicted in Figure $8 \mathrm{f}$. The loss of contact between the current collector and the coating reduces the adhesion and increases the impedance between electrode and current collector. The reduced contact and adhesion between electrode and current collector could lead to partial electrode delamination resulting in loss of electrode material. Consequently, the electrode capacity reduces and capacity fade is more severe. The $3 \mathrm{X}$ non-uniform coating has more interfacial regions than the $1 \mathrm{X}$ non-uniform coating has, and that difference might account for the faster capacity degradation in the electrode with the $3 \mathrm{X}$ defect.

\section{Conclusions:}

This study presented a systematic investigation on the effect of different types of electrode manufacturing defects in a positive lithium-ion battery electrode on its electrochemical performance . Although, the results reported in the manuscripts are based on full coin cell matrix, and the quantitative results in a larger format cells might be different. In addition, size of the defects may alter the rate of capacity degradation. However, the relative effects of the defects in the larger format cells might be similar to the effects that were observed in a full coin-cell matrix. The conclusions are:

i) Four different types of defects (agglomerates, pinholes, metal particle contaminants, and non-uniform coatings) were investigated. IR thermography was carried out as a QC or NDE method to detect the defects during coating in a slot-die coater.

ii) The electrode agglomerations and pinholes show lower coulombic efficiency at higher current density. The electrode pinholes, metal particle contaminants, and non-uniform coatings show poor rate capability, especially at higher rates. The capacity-fade tests in higher current densities reveal a faster capacity degradation in the electrodes having 
agglomerates. Furthermore, the rate of capacity degradation is even faster if there are more interfaces between coated and uncoated regions.

iii) Microstructural investigation on selective defective electrode matrix (agglomeration) revealed that the agglomerated region is mainly carbon-rich and that the agglomerations might decrease the electrochemical performance at higher-rate cycling. The poor performance in the non-uniform coatings with more coated-uncoated interface (the $3 \mathrm{X}$ specimens) is attributed due to the poor coating thickness in the interfacial lengths.

To summarize the relation between the questions asked in the introduction and the conclusions drawn, conclusion i answers the question i, conclusion ii answers questions ii, and iii, And conclusion iv answers the questions iv.

The findings in this research demonstrate the importance of monitoring and early detection of the electrode defects and flaws during LIB cathode manufacturing processes. Therefore, increased vigilance will reduce the scrap rate for electrodes and will minimize the cell rejection rate after fabrication and testing.

\section{Acknowledgments:}

This research at Oak Ridge National Laboratory, managed by UT Battelle, LLC, for the U.S. Department of Energy (DOE) under contract DE-AC05-00OR22725, was sponsored by the Office of Energy Efficiency and Renewable Energy for the Vehicle Technologies Office's Applied Battery Research Program (Program Managers: Peter Faguy and David Howell). Scanning Electron Microscopy experiments were conducted at the Center for Nanophase Materials Sciences, which is a DOE Office of Science User Facility. Debasish thanks Shawn Reeves for her help in collecting backscattered SEM images.

\section{References:}

[1] D. T. Danielson, U.S. Department of Energy-EV Everywhere grandchallenge blue print, http://energy.gov/sites/prod/files/2014/02/f8/eveverywhere_road_to_success.pdf, (2013). ( Acessed : January 2016). 
[2] D. Howell, U.S. Department of Energy (2012).

[3] J. M. Tarascon, M. Armand, Nature, 451 (2008) 652.

[4] M. S. Whittingham, Materials Research Bulletin, 33 (2008) 411.

[5] http://www.uscar.org/guest/teams/12/U-S-Advanced-Battery-Consortium. ( Acessed : January 2016).

[6] http://www.uscar.org/guest/article_view.php?articles_id=85. ( Acessed : January 2016).

[7] D. L. Wood III, J. Li, C. Daniel, Journal of Power Sources, 275 234-242.

[8] K.G. Gallagher, D. Dees, P. Nelson, PHEV Battery Cost Assessment, in: DOE

Annual Merit Review, May 9, (2011).

[9] J. Li, C. Daniel, D. L. Wood III, Journal of Power Sources, 196 (2011) 2452.

[10] Y. Xie1, W. Zhang, S. Gu, Y. Yan, Z.-F. Ma, Chinese Journal of Chemical Engineering, doi:10.1016/j.cjche.2015.07.013 (2015).

[11] A. Etiemble, N. Besnard, J. Adrien, P. Tran-Van, L. Gautier, B. Lestriez, E. Maire, Journal of Power Sources, 298 (2015) 285.

[12] http://www.purdue.edu/newsroom/releases/2013/Q2/new-technique-to-improve-qualitycontrol-of-lithium-ion-batteries.html, (2013). ( Acessed : January 2016).

[13] D. L. Wood III, J. Li, C. Daniel, Journal of Power Sources, 275 (2015) 234.

[14] J. Li, B. L. Armstrong, J. Kiggans, C. Daniel, D. L. Wood III, Langmuir, 28 (2012) 3783.

[15] D. Mohanty, J. Li, R. Born, L. C. Maxey, R. B. Dinwiddie, C. Daniel, D.L.W. III, Analytical Methods, 6 (2014) 674.

[16] http://www.abr.anl.gov/pdfs/2013_presentations/es164_wood_2013_p.pdf. ( Acessed : January 2016). 


\section{Figure Captions}

Figure 1: Optical and IR images of the defects of agglomerates (a, b), pinholes (c, d), metal particle contamination (e, f), and exaggerated non-uniform coating $(g, h)$. The emissivity values in the IR thermographs increase or decrease depending on the nature of the defects.

Figure 2: Comparison of rate capability and coulombic efficiency from the baseline NMC 532 electrodes and the electrodes with agglomerates $(a, b)$ and pinholes $(c, d)$.

Figure 3: Comparison of rate capability and coulombic efficiency from the baseline NMC 532 electrodes and electrodes with exaggerated non-uniform coating (a, b), Co metal contaminants (c, d), and Al foil contaminants (e, f).

Figure 4: Schematic of a regular shim set for a slot-die coater used for cathode coating (left) and schematic of the customized shim set used to apply an uneven coat of electrode slurry (right) (a). Optical image (b) and IR thermography image (c) showing the $1 \mathrm{X}$ and $3 \mathrm{X}$ defects. Comparison of rate capability and coulombic efficiency from the baseline NMC 532 electrode and an electrode with a $1 \mathrm{X}$ defect. Right ( $\mathrm{d}$ and e): Comparison of rate capability and coulombic efficiency of the NMC 532baseline electrode and an electrode with a $3 \mathrm{X}$ defect (f, and $\mathrm{g}$ ).

Figure 5: Comparison between gravimetric capacity-fading results for the baseline and defective NMC 532 electrodes at 2C and 5C current rates.

Figure 6: The gravimetric and full capacity before and after 200 charge-discharge cycles at 2C (a) and 5C (b) discharge rate. The capacity retention values after 200 cycles for baseline and defective electrodes at the $2 \mathrm{C}$ discharge rate and at the $5 \mathrm{C}$ discharged rate (c). 
Figure 7: Scanning electron micrographs and elemental EDS maps (Mn, Ni, Co, C, O) from (a) the baseline NMC 532 electrode and (b) the defective agglomerated electrode.

Figure 8: (a-c) Cross-sectional SEM images of the baseline NMC 532 electrodes, (d-g) crosssectional SEM images from an electrode having a non-uniform coating, and (h) SEM image from the electrode having a non-uniform coating. 


\section{Figures}

\section{Figure 1}

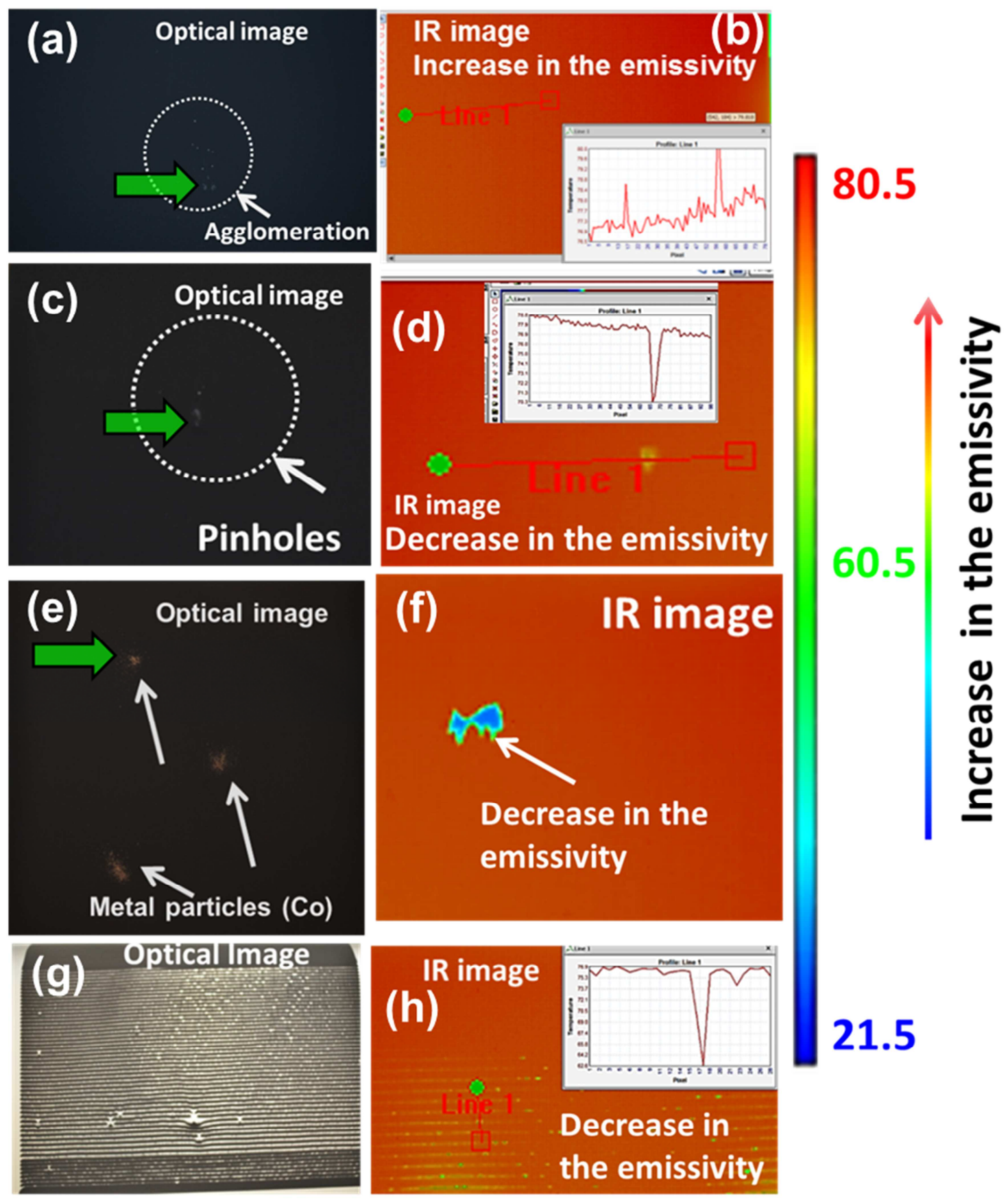


Figure 2:

Defect type: Agglomerates
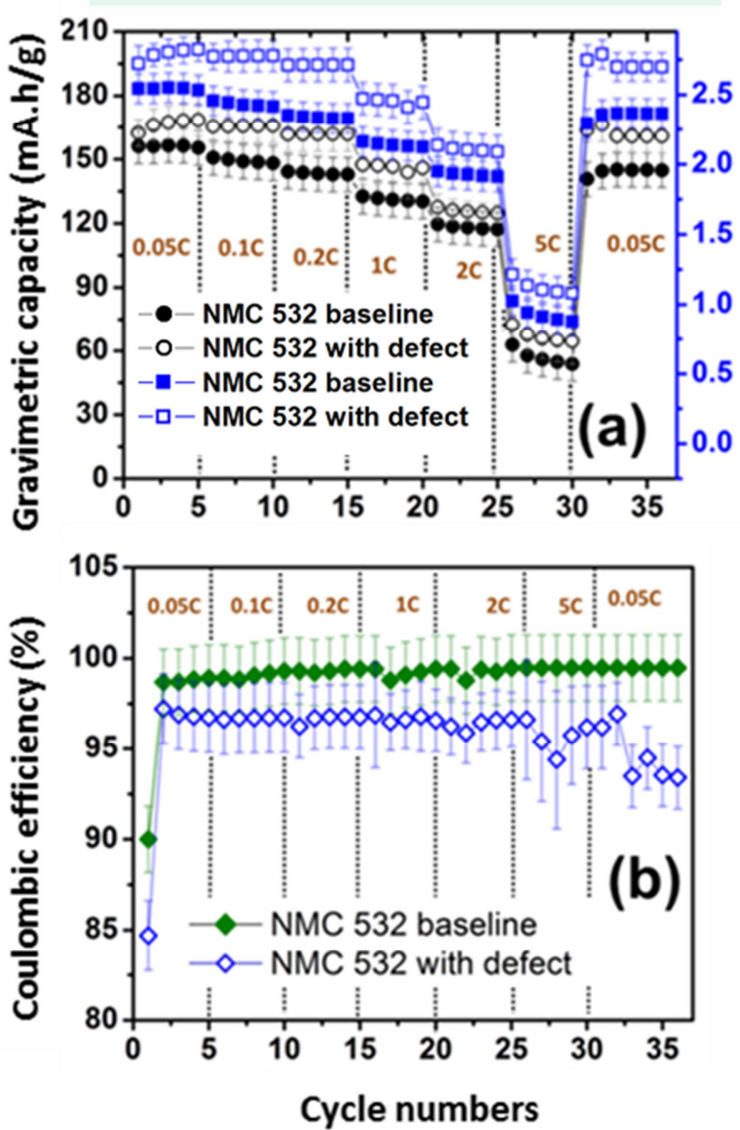

Defect type: Pinholes
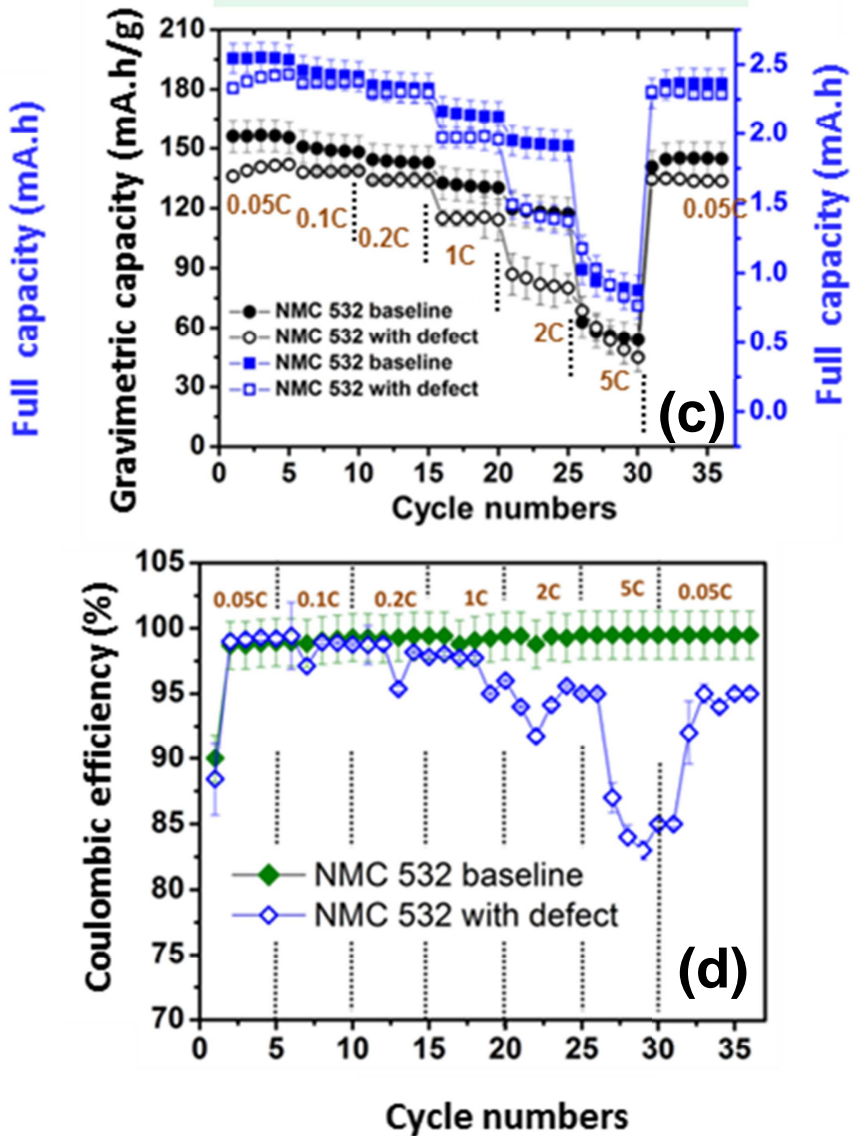
Figure 3:
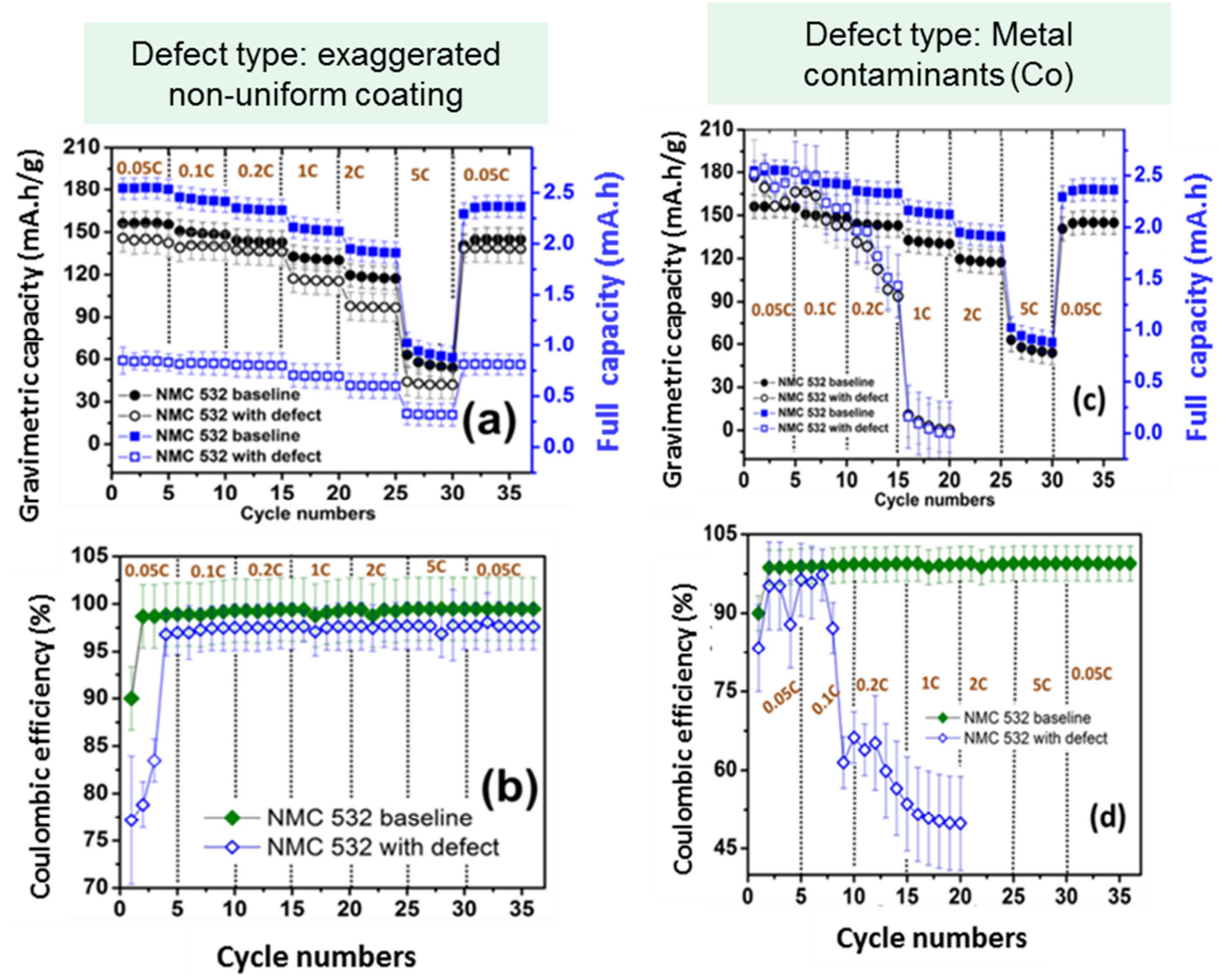
Figure 3
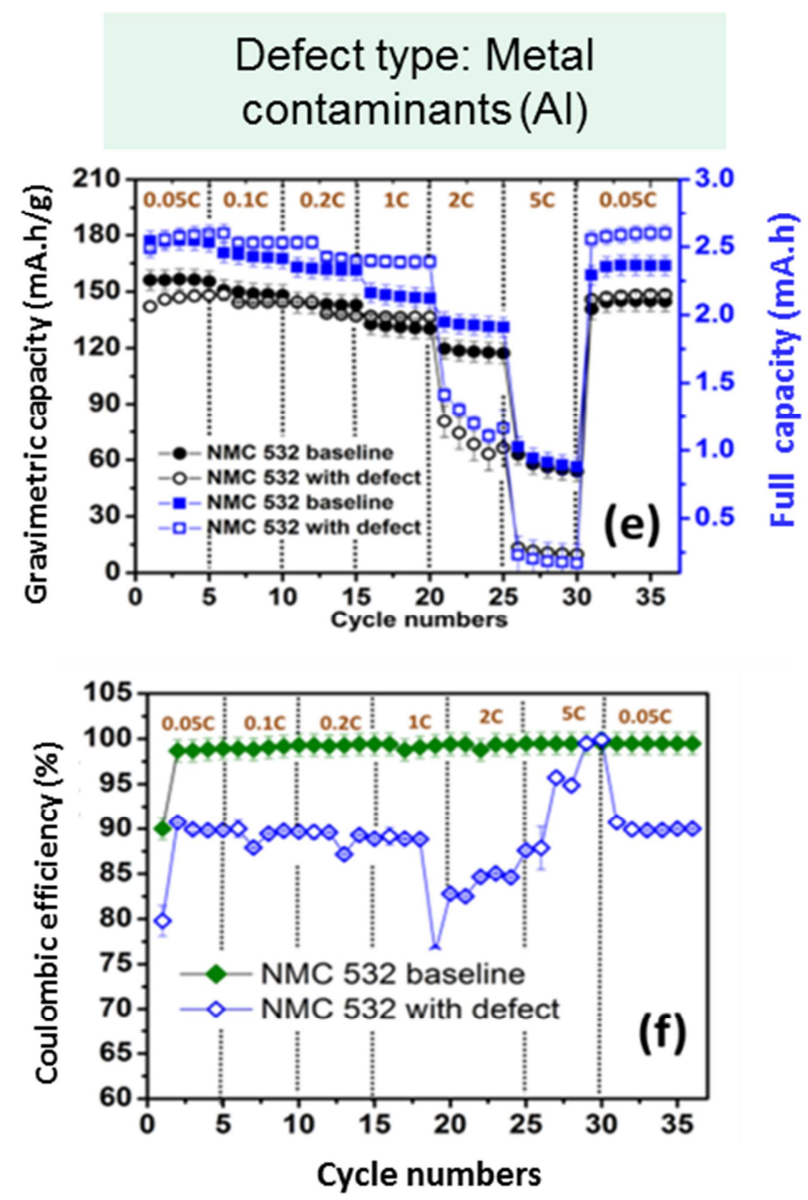

Figure 4:
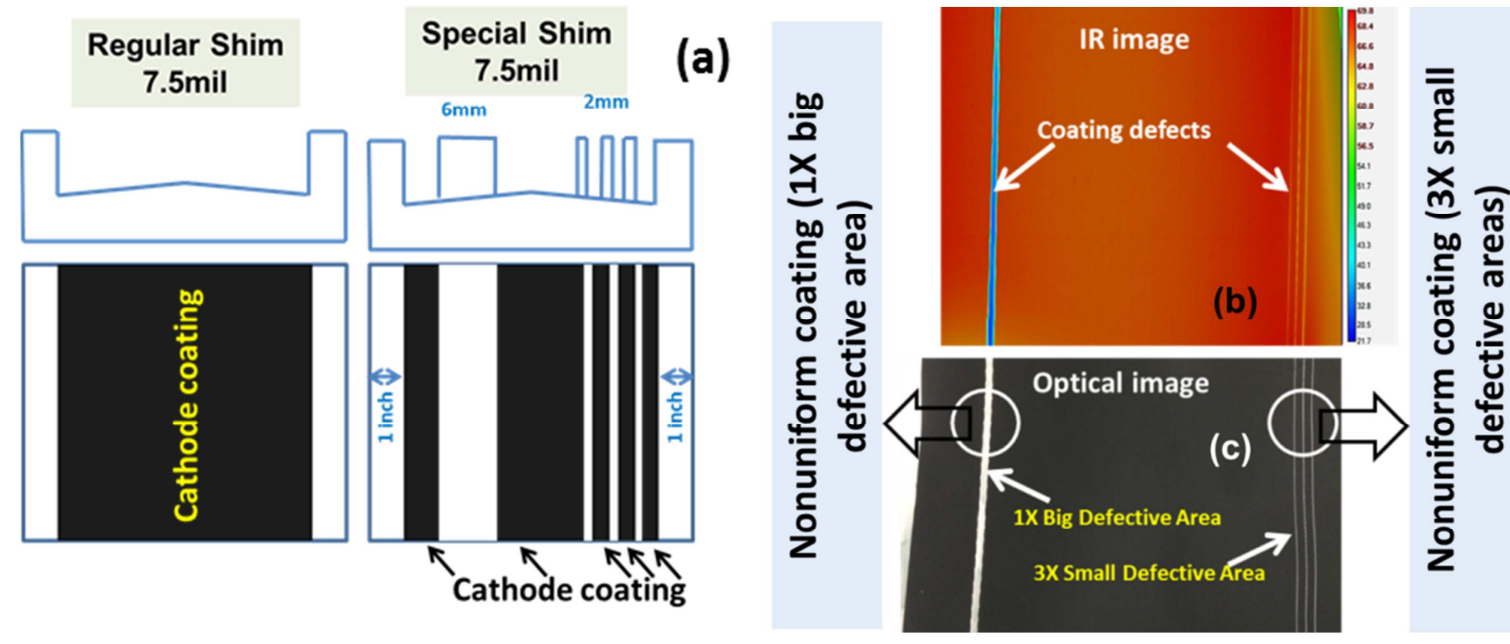


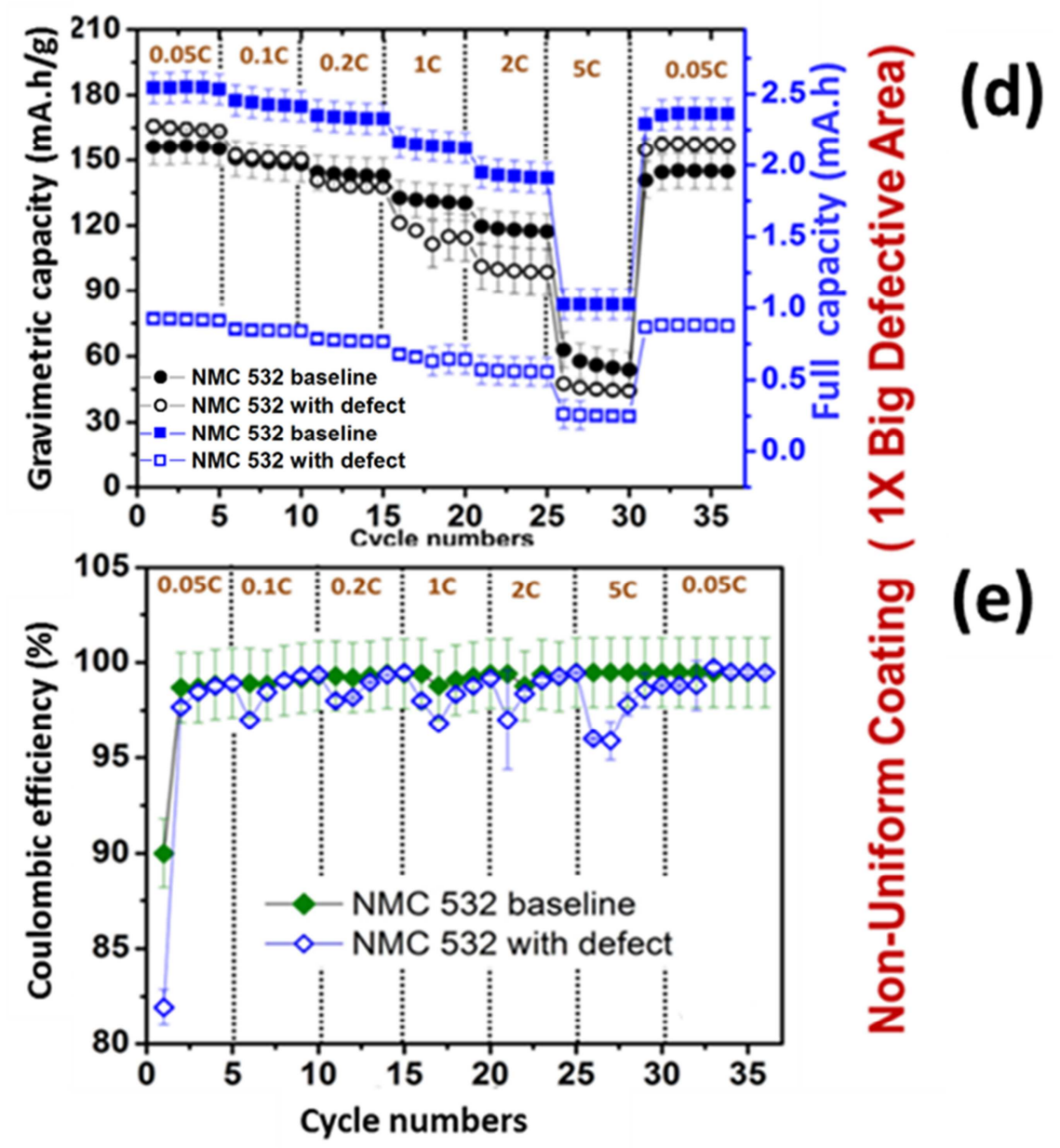




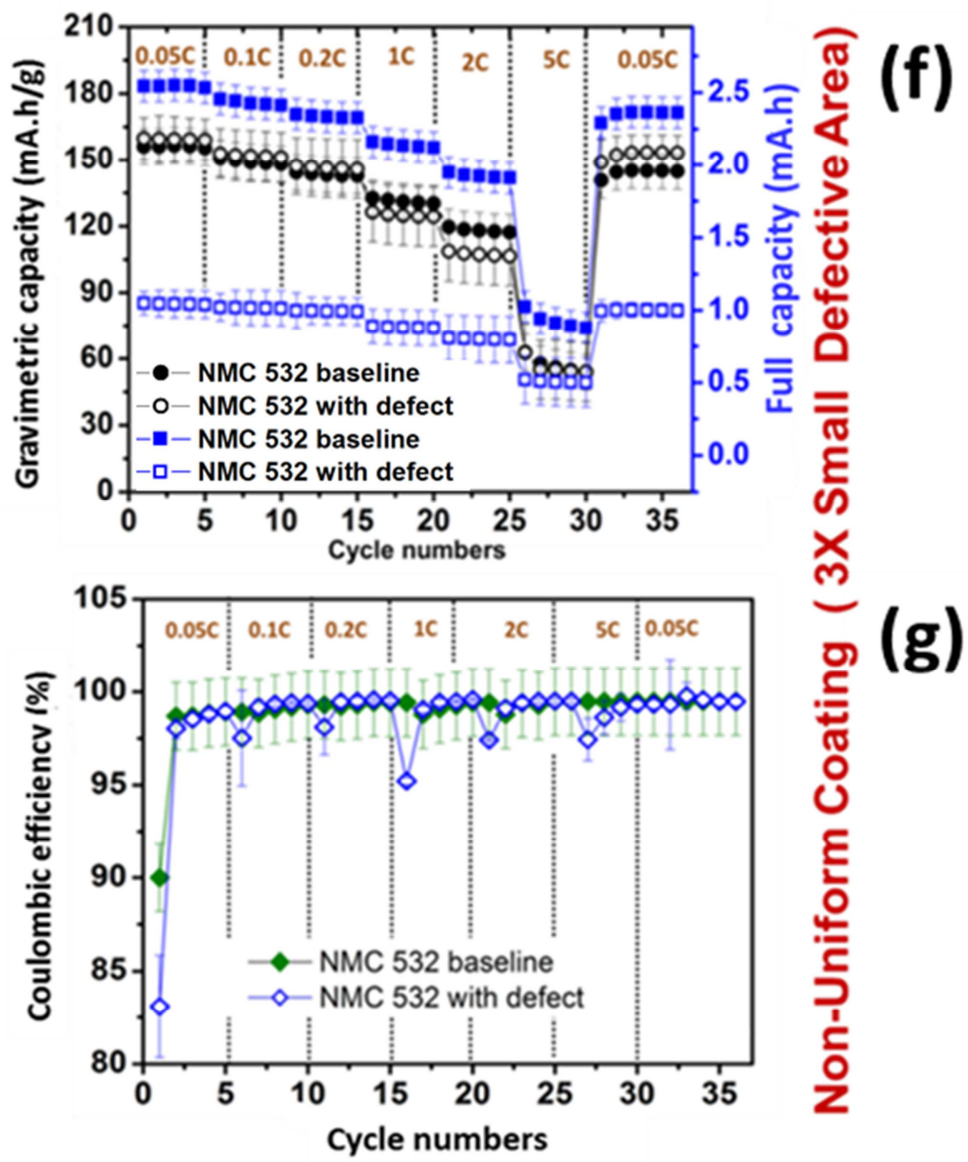


Figure 5:
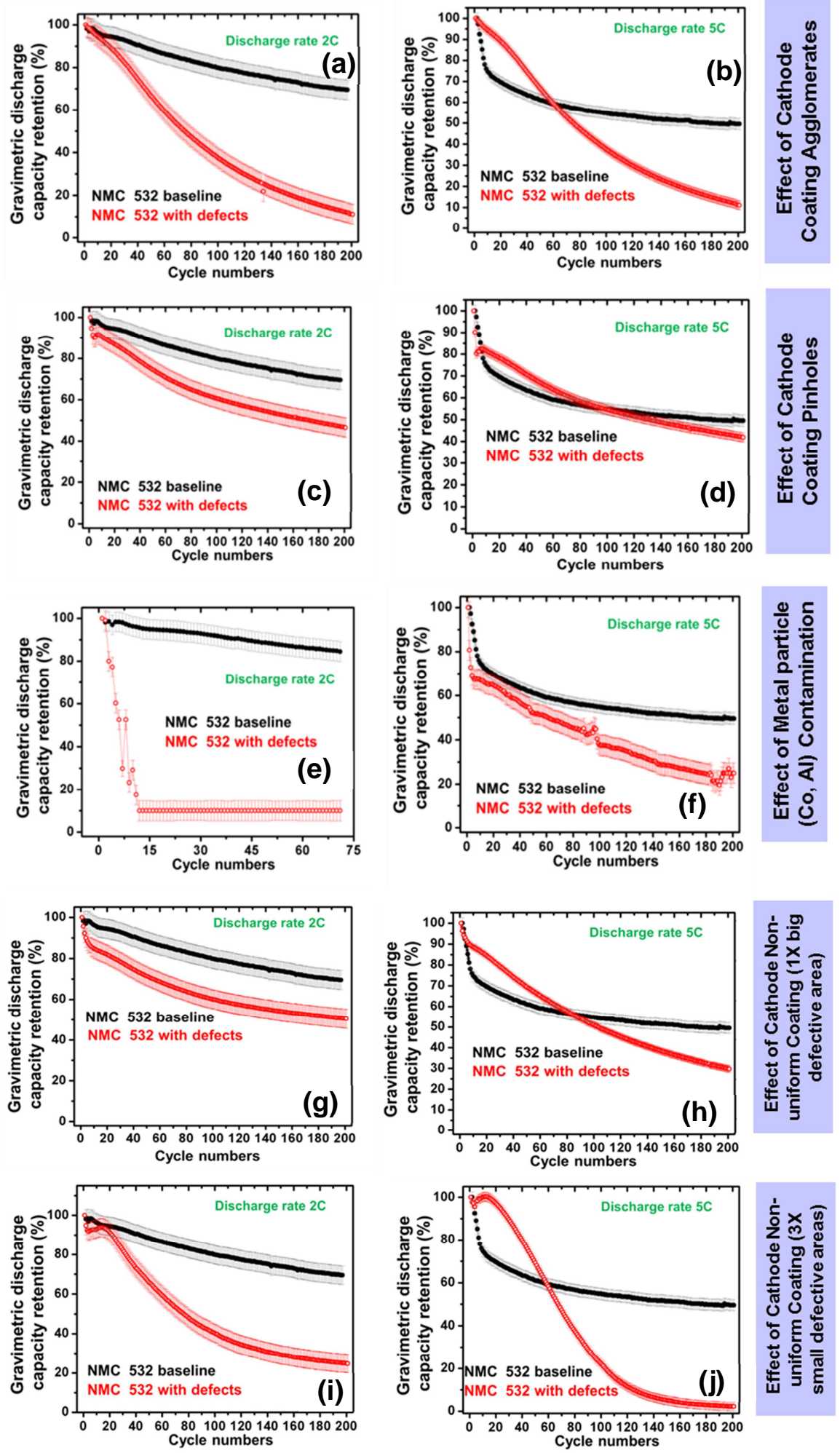
Figure 6:
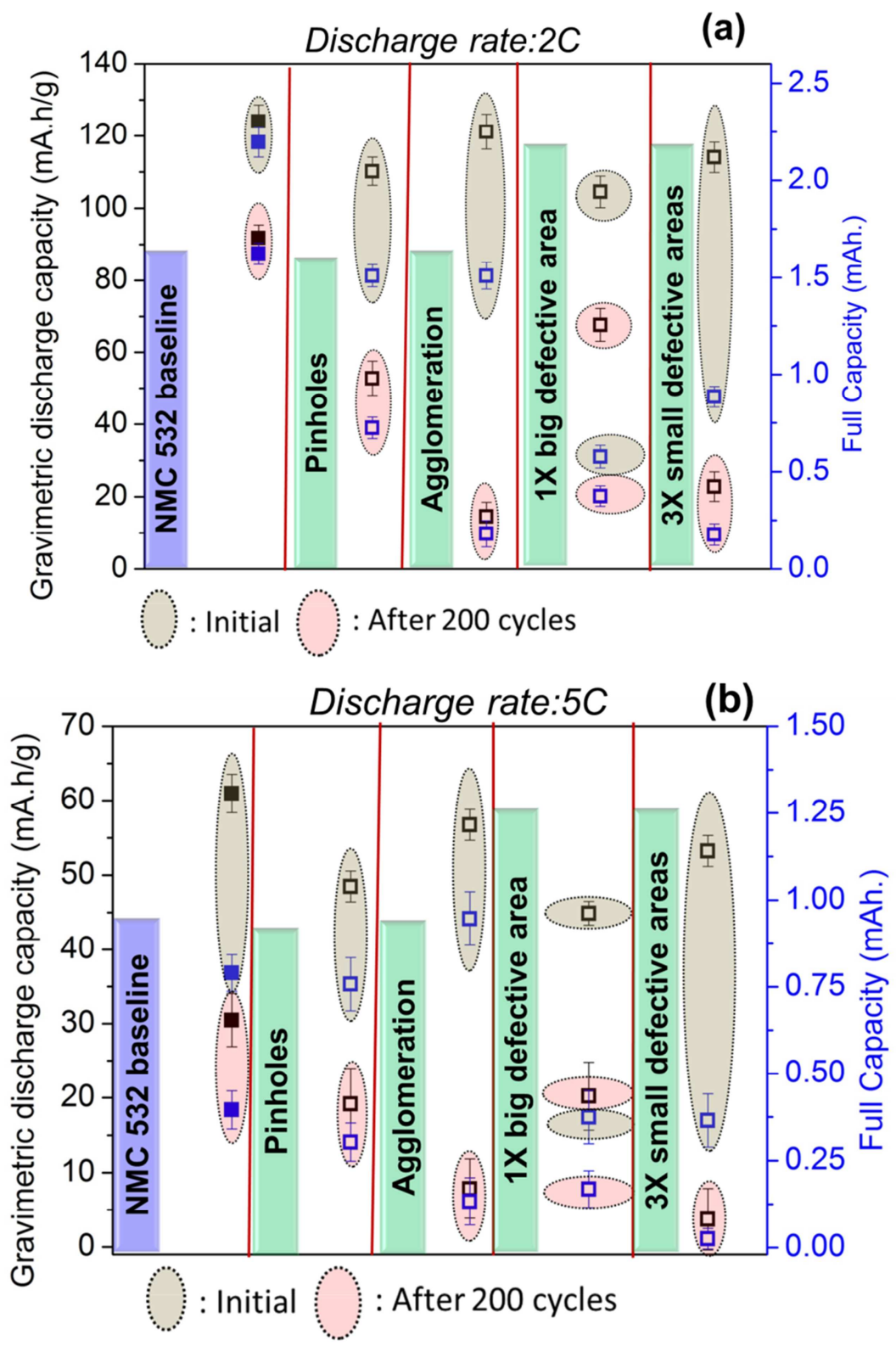


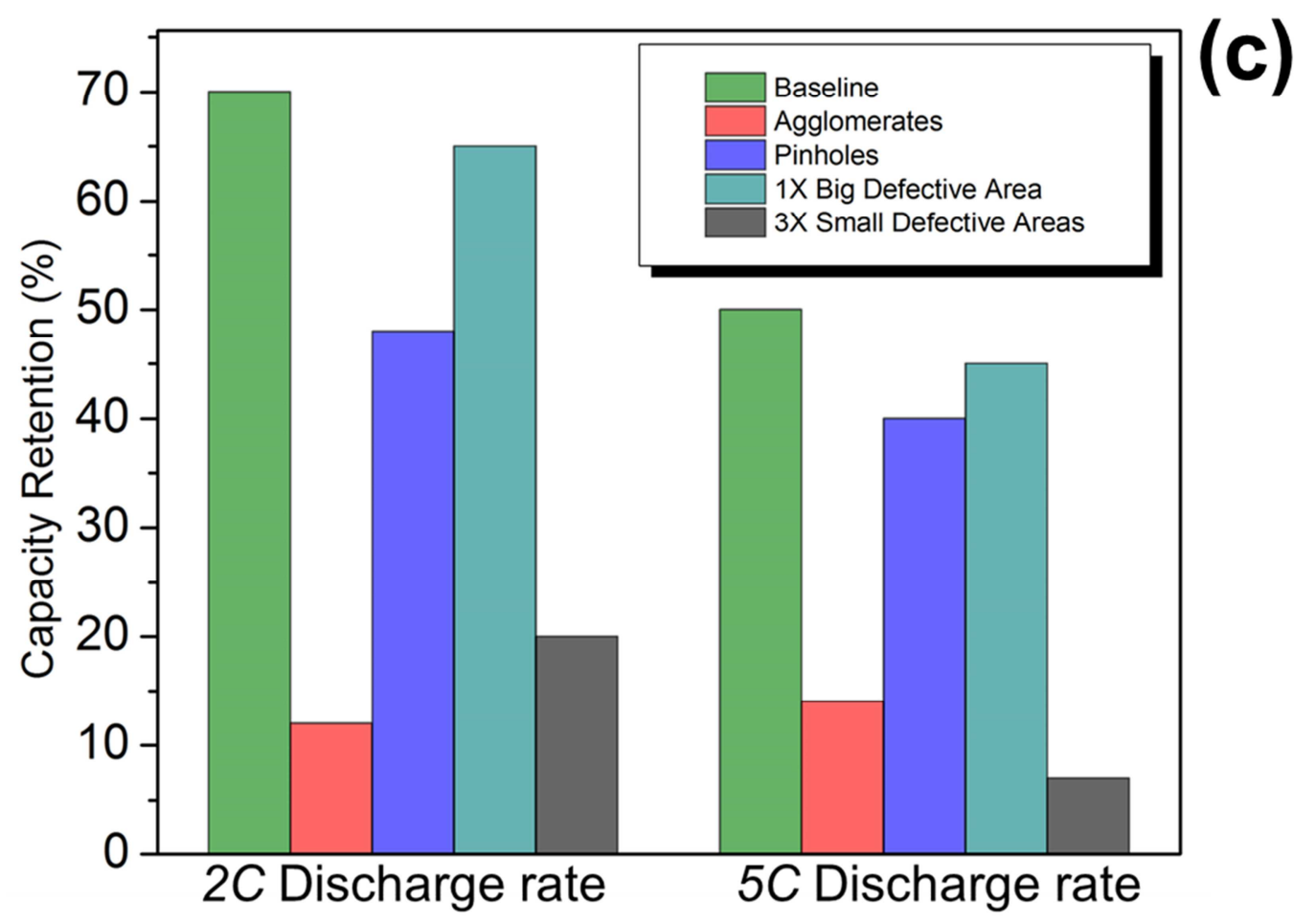


Figure 7:
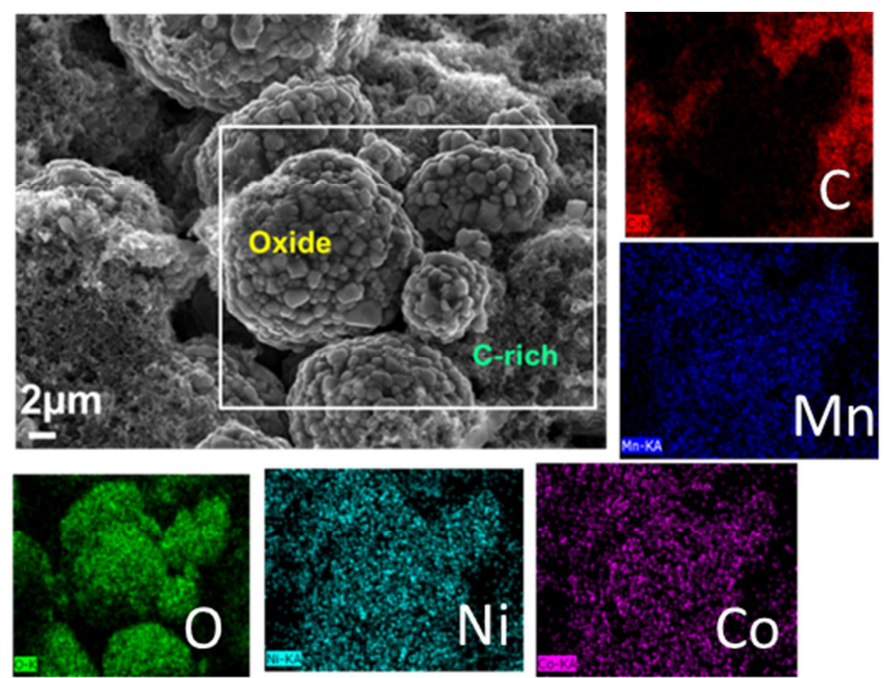

(a)

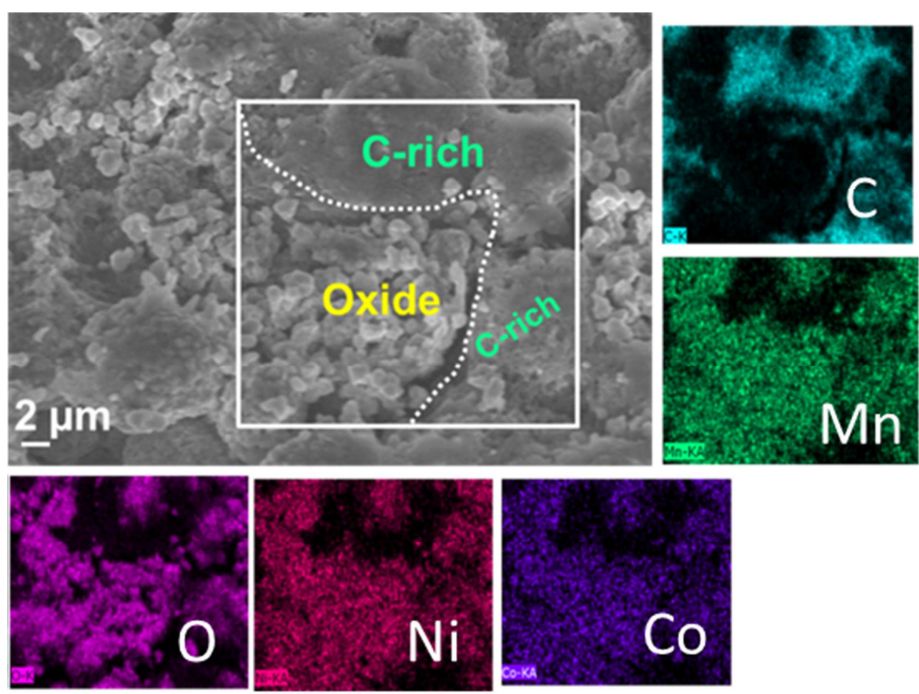

(b) 
Figure 8:

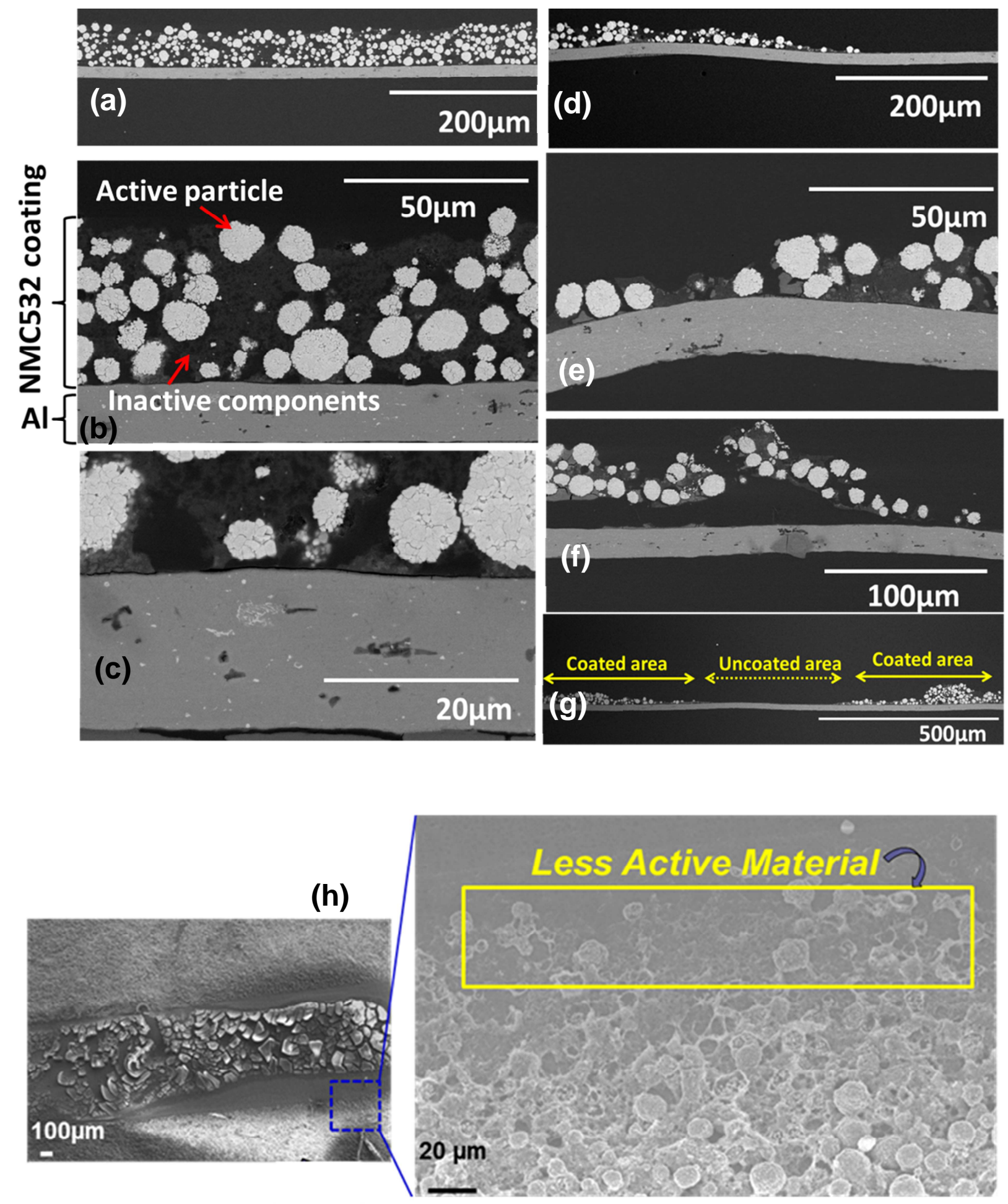

